\title{
A Strategy for Adopting Server Virtualization in the Public Sector: NIPA Computer Center
}

\author{
Jong-Cheol Song ${ }^{1}$, Jee-Woong Ryu ${ }^{1,2}$, and Byung-Joo Moon ${ }^{1}$, Hoe-Kyung Jung ${ }^{2 *}$, Member, KIICE \\ ${ }^{1}$ Information Service Team, National IT Industry Promotion Agency, Daejeon 305-348, Korea \\ ${ }^{2}$ Department of Computer Engineering, Paichai University, Daejeon 302-735, Korea
}

\begin{abstract}
Many public organizations have been adopting and operating various servers. These servers run on Windows, Unix, and Linux operating systems that generally use less than $10 \%$ of their capacity. For migrating a public organization to cloud computing, we must first virtualize the server environment. This article proposes a strategy for server virtualization that the National IT Industry Promotion Agency (NIPA) has done and describes the effects of a public organization migrating to cloud computing. The NIPA Computer Center planned an effective virtualization migration on various servers. This project of virtualization migration was conducted with the existing policy of separate x86 servers and Unix servers. There are three popular approaches to server virtualization: a virtual machine model, a paravirtual machine model, and virtualization at the operating system layer. We selected a VMware solution that uses the virtual machine model. We selected servers for virtualization in the following manner. Servers were chosen that had the highest rate of service usage and CPU usage and had been operating for five years or more. However, we excluded servers that require $80 \%$ or greater rates of CPU usage. After adopting the server virtualization technique, we consolidated 32 servers into 3 servers. Virtualization is a technology that can provide benefits in these areas: server consolidation and optimization, infrastructure cost reduction and improved operational flexibility, and implementation of a dual computing environment.
\end{abstract}

Index Terms: Server virtualization, Hypervisor, Virtualization strategy

\section{INTRODUCTION}

The rapid evolution of computing and communications technology offers unprecedented opportunities for the public sector.

In an era of explosive data growth and flat budgets, organizations must evaluate potential technology purchases not only on their technical merits but on their economic merits. All virtualization can deliver significant benefits to the organization by improving computing utilization and overall efficiency while reducing costs and complexity.

Virtualization is a term that is often applied to a wide range of technologies. Essentially, virtualization technology decouples the software used in the realm from the hardware. In the area of server virtualization, this means that multiple server environments (Windows, Linux, etc.) can be housed on a single piece of physical hardware [1].

For migrating a public organization to cloud computing, we first have to virtualize the server environment, which brings two key issues. One is how to optimize and consolidate a largescale heterogeneous computing system. The other is how to realize the virtualization server environment.

This paper, therefore, focuses on how to effectively build the virtualization servers for public organizations. It deals with the

Received 07 December 2011, Revised 27 January 2012, Accepted 05 February 2012

*Corresponding Author E-mail: hkjung@pcu.ac.kr 
issues that resulted from adopting virtualization servers in public organizations. It considers virtual servers that have been implemented in National IT Industry Promotion Agency (NIPA). Finally, it reviews the benefits of server virtualization and suggests key issues for future research.

\section{CASE STUDY}

How does virtualization work? Generally, server virtualization solutions work by introducing a thin layer over the physical server. This layer partitions the physical server into separate areas that the virtual servers then run on. Computing resources from the underlying server are viewed as a pool of resources that can then be shared among the virtual machines sitting on top. With the exception of sharing computing resources, each virtual server acts as its own entity; problems with an application on one server do not affect other virtual machines on that same physical server [1].

There are three popular approaches to server virtualization: the virtual machine model, the paravirtual machine model, and virtualization at the operating system layer.

The hypervisor is called a virtual machine monitor (VMM). It validates all the guest-issued CPU instructions and manages any executed code that requires additional privileges. VMware and Microsoft Virtual Server both use the virtual machine model [2]. The paravirtual machine (PVM) model is also based on the host/guest paradigm and it uses a virtual machine monitor, too. In the PVM model, however, the VMM actually modifies the guest operating system's code. This modification is called porting, which supports the VMM so it can utilize privileged system calls sparingly. Like virtual machines, PVMs are capable of running multiple operating systems (OS). Virtualization at the OS level works a little differently. It is not based on the host/guest paradigm [3].

\section{EFFECTIVE SERVER VIRTUALIZATION STRATEGY}

There are many public organizations that have been adopting and operating various servers. These servers run on Windows, Unix, and Linux operating systems and generally use less than $10 \%$ of their capacity.

There are five types of OSs in the NIPA e-Cube Center. Windows has the highest share of adoption of any server operating system. The adoption share estimates for Linux are $22 \%$ and for Solaris are $21 \%$. NIPA had been adopting and operating various servers. These servers had been running on Windows, Unix, and Linux operating systems.
These servers generally have used less $10 \%$ of their capacity in less important service. Fig. 1 indicates various servers in the NIPA e-Cube Center.

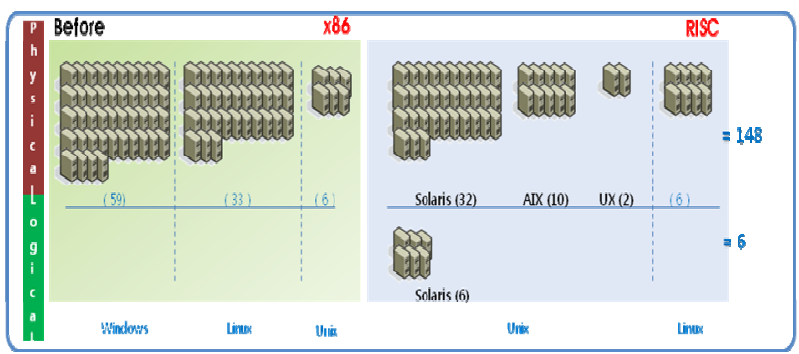

Fig. 1. Ratio of operating system.

If they can be consolidated onto a smaller number of servers using virtualization technology, we can increase the space utilization efficiency in a datacenter and boost hardware utilization to between 50 and 80\% [4].

The following server virtualization strategy describes the methods one should consider when for embarking on a server virtualization project in a public organization.

First, the server machines were divided into X86 servers and non-X86 servers. Second, the X86 servers were virtualized and consolidated.

Third, the non-X86 servers were virtualized. That is, the IBM, HP, and Sun servers were virtualized with a different vendor's virtual tool.

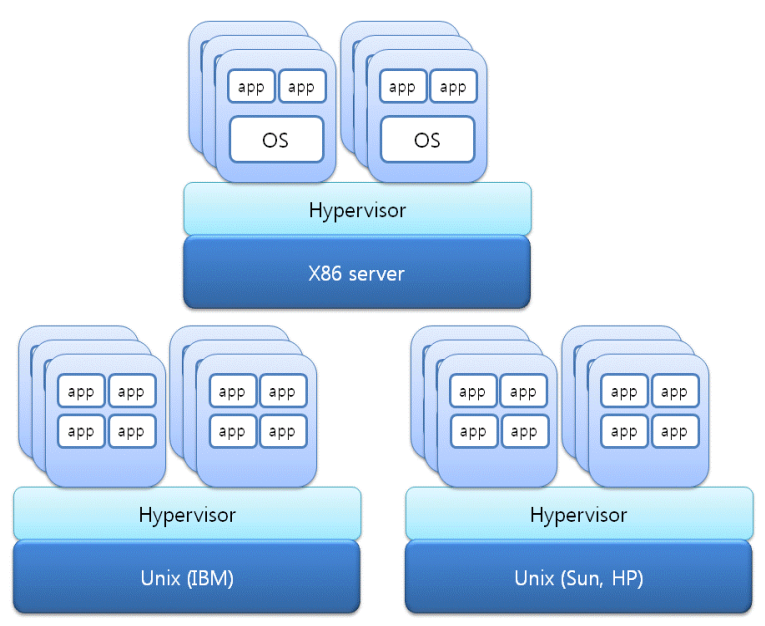

Fig. 2. Virtualization strategy for servers.

The X86 server virtualization tool (hypervisor) allows quicker and easier virtualization than for Unix servers. Fig. 2 indicates virtualization strategy by type of server [3]. 


\section{ADOPTING SERVER VIRTUALIZATION STRATEGY}

\section{A. Building the Virtualization Server Environment}

The NIPA Computer Center designed an effective virtualization migration of its various servers. This virtualization migration plan kept x86 servers and Unix servers separate. We adopted the virtual machine model. VMware was used as the virtual machine model. We used the VMware solution on X86 servers. However, for the Unix servers, we plan to use each vendor's virtual tool. We selected the X86 servers for virtualization using the following criteria: 1 ) servers that had a low rate of service and infrequent usage, 2) servers that had been operating for five years or more. In other words, older servers, to avoid upgrade costs.

There are multiple factors to consider when evaluating and selecting a server in a server virtualization plan. We excluded the servers for virtualization according to the following criteria: 1) servers with $80 \%$ or greater rates of average CPU usage. For example, $10 \mathrm{GHz}$ or more of average CPU usage, 16 GB or more of memory usage, 200 Mbps or more of network bandwidth usage, or $1 \mathrm{~TB}$ or more disk of disk usage, 2) servers with special devices, such as a fax card or CAD, tape drive, etc. Virtualization solutions cannot support these devices, 3) applications with a real-time service or mass transaction service function, 4) software that managed licenses into hardware lock-down (serial port, parallel port, and USB port) were excluded, because these servers disturb effective usage of the server's resources. NIPA's servers were virtualized with this server virtualization strategy. We implemented server virtualization with the VMware solution. Figs. 3-5 describe the implementation of the virtualization servers at NIPA.

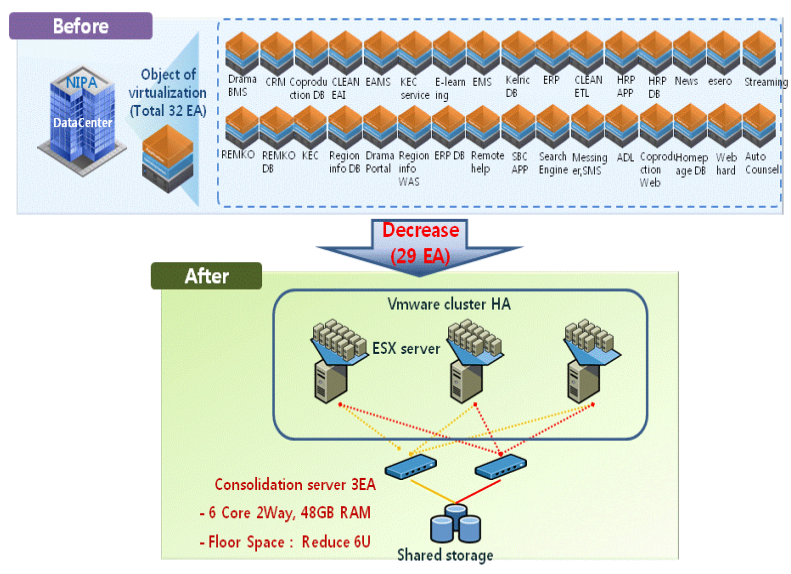

Fig. 3. Implementation of virtualization servers.

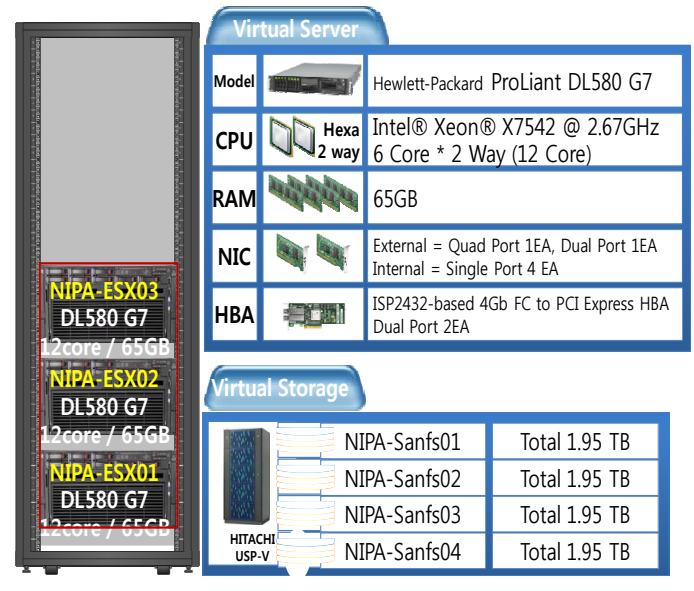

Fig. 4. Virtualization servers and storage.

The NIPA Computer Center implemented the server virtualization environment and eliminated 29 physical servers in an effective virtualization migration on the various servers.

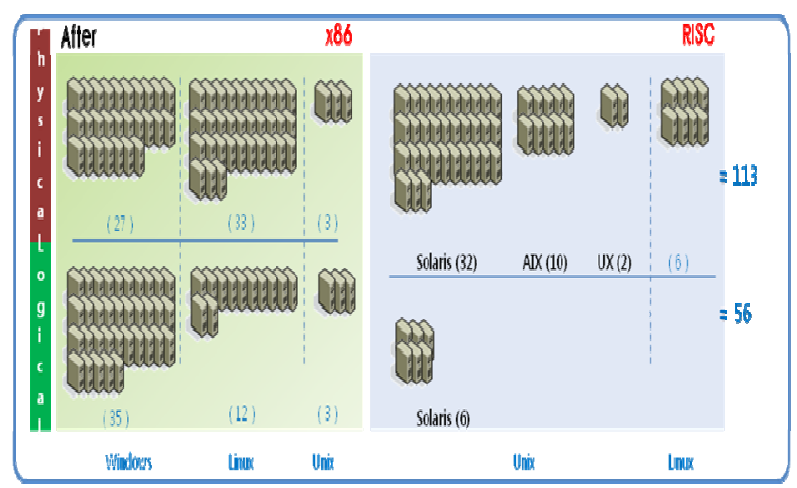

Fig. 5. Server configuration after server virtualization.

\section{B. Benefit of Server Virtualization}

We took advantage of the following: 1) server consolidation and optimization: we estimated that distributed physical servers generally use only $5 \%$ of their capacity, so by virtualizing those server environments, we can boost hardware utilization by $50 \%$; that is a $10: 1$ consolidation ratio [5]. As a result, we can reduce hardware maintenance costs by reducing the number of physical servers; 2) increasing the space utilization efficiency: virtualizing servers decreases the amount of floor space that we must use. Before virtualizing servers, $128 \mathrm{U}$ was used for floor space but after virtualizing the servers, $12 \mathrm{U}$ was used as floor space. That's a 10:1 reduction ratio. Table 1 demonstrates the advantages of server virtualization; 3) hardware cost: because virtualization allows for greater 
utilization of existing resources, fewer physical servers are required, saving money both on upfront hardware costs and on maintenance costs [6]. Virtualizing servers reduces the time it takes to provision new servers and maintenance servers by up to 25\%; Flexibility and dual system: Because virtualization allows for the quick creation of different operating system environments, it is easy to run legacy applications alongside new versions, migrate applications to new environments, and restore systems in post-disaster scenarios. We can operate dual systems.

Table 1. Increasing server operation efficiency in National IT Industry Promotion Agency

\begin{tabular}{lcc}
\hline & Before (min) & After (min) \\
\hline System installation & 250 & 0 \\
Operating system installation & 170 & 80 \\
Operating system patch & 90 & 20 \\
On and off & 30 & 10 \\
\hline
\end{tabular}
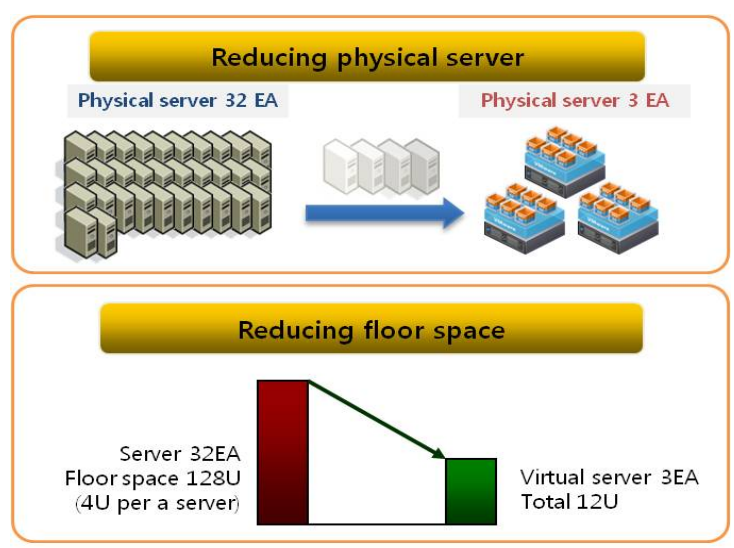

Increasing usability and adopting HA

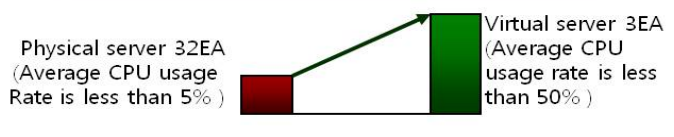

Fig. 6. Advantages of National IT Industry Promotion Agency's sever virtualization.

\section{CONCLUSIONS}

This paper proposes a strategy for server virtualization that NIPA applied to migrating to cloud computing. Two key issues arise in regard to the virtualization of servers in public organizations. One is how to optimize and consolidate large-scale heterogeneous computing systems. The other issue is how to realize a virtualized server environment. This virtualization migration plan kept X86 servers and Unix servers separate. We proposed a solution for the virtualization issues of X86 servers. We implemented a server virtualization environment.

We selected the X86 servers that were infrequently used or had been operating five or more years. However, we excluded servers that frequently use the CPU, that have a special device, or that have a real-time service function. Virtualization is a technology that can provide benefits in these areas: server consolidation and optimization, infrastructure cost reduction and space utilization, improved operational flexibility, and implementation of dual computing environments.

\section{REFERENCES}

[1] IT management. Server virtualization FAQ [Internet]. Available: http://www.itmanagement.com/faq/server-virtualization/.

[2] Hewlett Packard com. Server virtualization - overview. [Internet]. Available: http://www.hp.com/sbso/productivity/howto/it_server_ virtualization/server-virtualization.pdf.

[3] TechTarget. SearchServierVirtualization - definition server virtualization. [Internet]. Available: http://searchservervirtuali zation.techtarget.com/definition/server-virtualization.

[4] Vmware. Virtualization basics [Internet]. Available: www.vmware.com/virtualization/virtual-infrastructure.html.

[5] Vmware. Server consolidation [Internet]. Available: http://www.vmware.com/solutions/consolidation.

[6] ITword. Top 5 benefits of server virtualization [Internet]. Available: http://www.itworld.com/nls_windowsserver050411

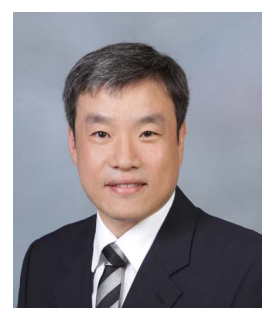

Jong-Cheol Song

He received a B.S degree from the Department of Computer Engineering from Kwangwoon University, Korea, in 1999, and he received a Ph.D. degree from the Department of Computer Engineering of Paichai University, Korea, in 2007. From 1999 to 2003, he worked for ETRI as a researcher. Since 2003, he has worked in the Information Service Team at the National IT Industry Promotion Agency (NIPA), where he now works as a deputy director. His current research interests include the semantic web. 


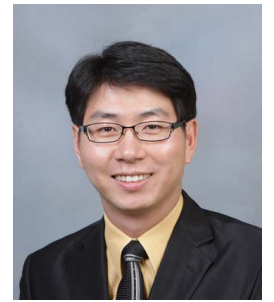

\section{Jee-woong Ryu}

He received a Master's degree from the Department of Information Technology of ICU, Korea, in 2003. From 2003 to 2009, he worked for IITA as a researcher. Since 2009, he has worked in the Information Service Team at the National IT Information Promotion Agency (NIPA), where he works as a reseacher. His current research interests are cloud computing, virtual desktop infrastructure, and PMS.

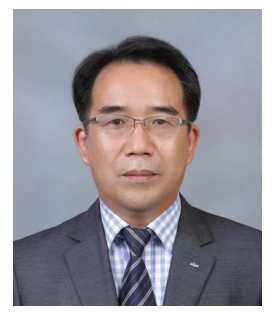

\section{Byung-Joo Moon}

He received the B.S degree from the Department of Computer Science of Chungbuk University, Korea in 2000. From 1991 to 2003, he worked for ETRI as a senior researcher. Since 2003, he has worked in the Information Service Team at the National IT Industry Promotion Agency (NIPA), where he now works as a team leader. His current research interests include web 2.0 , and information clustering.

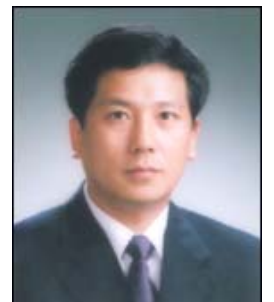

\section{Hoe-Kyung Jung}

He received the B.S degree in 1987 and $\mathrm{Ph}$. D. degree in 1993 from the Department of Computer Engineering of Kwangwoon University, Korea. From 1994 to 2005, he worked for ETRI as a researcher. Since 1997, he has worked in the department of Computer Engineering at Paichai University, where he now works as a professor. His current research interests include multimedia document architecture modeling, information processing, information retrieval, and databases. 